\title{
Phase structure of QCD for general number of flavors *
}

\author{
Y. Iwasakia, ${ }^{a, b}$ K. Kanaya, ${ }^{a, b}$ S. Kaya, S. Sakai, and T. Yoshiéa,b \\ ${ }^{a}$ Center for Computational Physics, University of Tsukuba, Ibaraki 305, Japan \\ ${ }^{\text {b}}$ Institute of Physics, University of Tsukuba, Ibaraki 305, Japan \\ ${ }^{\mathrm{c}}$ Faculty of Education, Yamagata University, Yamagata 990, Japan
}

We investigate and elucidate the phase structure of QCD for general number of flavors $N_{F}$ with Wilson quarks, varying $N_{F}$ from 2 up to 300 . Based on numerical results combined with the result of the perturbation theory we propose the following picture: When $N_{F} \geq 17$, there is only a trivial fixed point and therefore the theory in the continuum limit is trivial. On the other hand, when $16 \geq N_{F} \geq 7$, there is a non-trivial fixed point and therefore the theory is non-trivial with anomalous dimensions, however, without quark confinement. Theories which satisfy both quark confinement and spontaneous chiral symmetry breaking in the continuum limit exist only for $N_{F} \leq 6$. We also discuss the structure of the deconfining phase at finite temperatures for the small number of flavors such as $N_{F}=2$ and 3 , through a systematic study of it for general number of flavors.

\section{Introduction}

In our previous work [1], it was shown that in the strong coupling limit, when the number of flavors $N_{F}$ is greater than or equal to 7 , quarks are deconfined and chiral symmetry is restored at zero temperature, if the quark mass is lighter than a critical value. On the other hand, it is wellknown that when $N_{F}$ exceeds $16 \frac{1}{2}$, asymptotic freedom is lost.

In this work we investigate the problem of what is the condition on the number of flavors for quark confinement and spontaneous chiral symmetry breaking in the continuum limit, using the Wilson quark action and the standard one-plaquette gauge action. We first clarify the phase structure at zero temperature for general number of flavors, in particular, for $N_{F} \geq 7$. When the phase diagram becomes clear, we are able to see what kind of continuum limit exists and eventually answer the problem given above.

In order to reveal the structure of the deconfining phase, we increase $N_{F}$ up to 300 , because the region of the deconfining phase at zero temperature becomes larger with larger $N_{F}$, and consequently the structure becomes clear, as shown be-

\footnotetext{
*Presented by K. Kanaya and S. Kaya. at Lattice 96, St.
} Louis, USA. low. Then combining all data including the case of small $N_{F}$, we are able to conjecture the phase structure for general number of flavors. Through this systematic study of the deconfining phase for general number of flavors, we are also able to understand the behavior of the quark mass $m_{q}$ (defined through an axial-vector Ward identity) and $m_{\pi}^{2}$ as functions of $1 / K$ in the deconfining phase at finite temperatures in particular at small $\beta$ for small number of flavors such as $N_{F}=2$ and 3 .

\section{Simulation parameters}

The lattice sizes are $8^{2} \times 10 \times N_{t}\left(N_{t}=4,6\right.$ or 8), $16^{2} \times 24 \times N_{t}\left(N_{t}=16\right)$ and $18^{2} \times 24 \times N_{t}$ $\left(N_{t}=18\right)$. We use an anti-periodic boundary condition for quarks in the $t$ direction and periodic boundary conditions otherwise. When the hadron spectrum is calculated, the lattice is duplicated in the direction of lattice size 10 for $N_{t} \leq 8$, which we call the $z$ direction. We call the pion screening mass simply the pion mass and similarly for the quark mass. The current quark mass $m_{q}$ is defined by an axial-vector Ward identity [2, 3].

We use the hybrid $\mathrm{R}$ algorithm for the quark matrix inversion. As $N_{F}$ increases we have to decrease $\Delta \tau$, such as $\Delta \tau=0.0025$ for $N_{F}=240$, to reduce $O\left(\Delta \tau^{2}\right)$ errors. We have checked that 


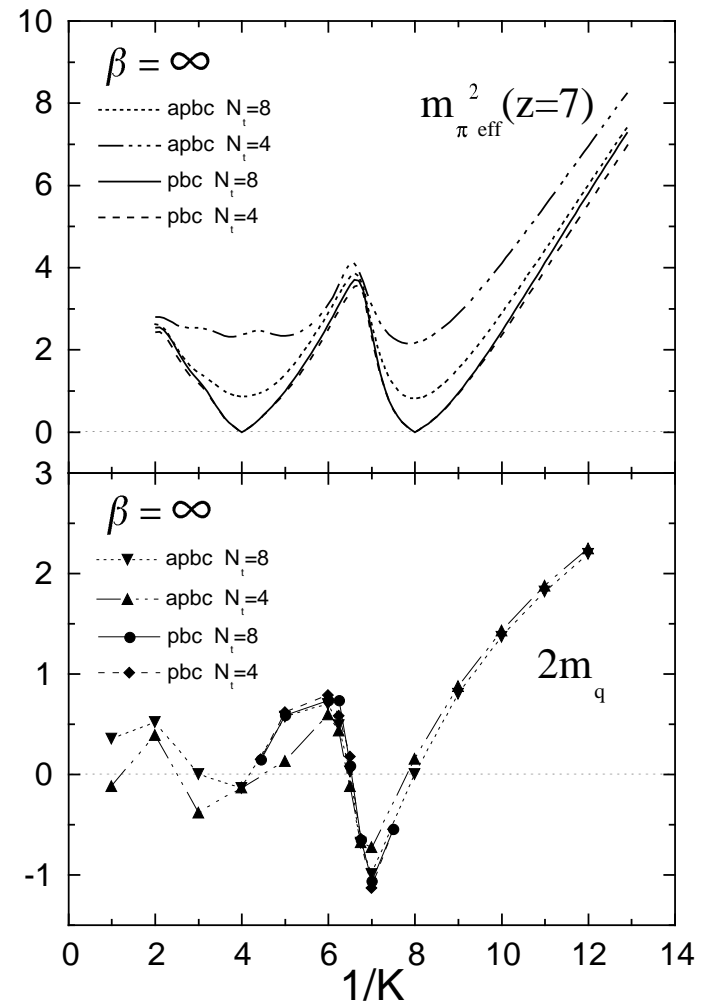

Figure 1. The effective pion mass obtained at $\mathrm{z}=7$ and the current quark mass for a free quark versus $1 / K$ for $N_{t}=4$ and 8 with periodic and antiperiodic boundary conditions on $40^{3} \times N_{t}$ lattices.

the errors are sufficiently small selecting typical cases.

\section{Free case: $\beta=\infty$}

We first investigate the free case, because the free case is important as a reference to understand the structure of the deconfining phase. We will show later that the $1 / K$ dependence of the pion mass in the deconfining phase is quite similar to that of free quarks for any $N_{F}$ and $\beta$.

Because the Wilson formalism lifts the doublers by the Wilson term, there is only one pole in the free quark propagator at $1 / K \simeq 8$. However, there is reminiscence of doublers.

In Fig. 11 we plot the effective pion mass obtained at $z=7$ from the pion propagator. The four cases shown there correspond to those for
$N_{t}=4$ and 8 with an anti-periodic or the periodic boundary condition in the $t$ direction.

In the case of the periodic boundary condition, there are almost no differences between the $N_{t}=$ 4 and 8 cases. The vanishing of $m_{q}$ at $1 / K=8$ and the behavior of $m_{\pi}^{2}$ for $1 / K \geq 8$ roughly given by $m_{\pi}^{2} \sim(1 / K-8)$ are all as naively expected.

For $1 / K \leq 8$, the behavior of $m_{\pi}^{2}$ is somewhat complicated. The vanishing of $m_{\pi}$ at $1 / K=4$ is due to doublers; one of momenta in spatial direction is $\pi$. At $1 / K \simeq 6$, the momentum-energy (dispersion) relation is completely opposite to the usual one: The energy is a decreasing function of the magnitude of momentum for the case of equal momenta in three spatial dimensions. The energy becomes infinity at zero momenta like the infinite mass case, while it is finite for other modes of momenta. Therefore the appearance of the peak of $m_{\pi}$ at $1 / K \simeq 6$ is due to a superposition of contributions from several modes of momenta. The behavior of $m_{q}$ defined through the axial-vector Ward identity is also complicated at $1 / K \leq 8$ due to doublers. In the following, we refer to the rightmost $m_{q}=0$ state (at $1 / K=8$ for $\beta=\infty$ ) as simply the massless quark, or $m_{q}=0$, distinguished from the other $m_{q}=0$ states, unless otherwise stated.

When an anti-periodic boundary condition is imposed, the position as well as the height of the peak of $m_{\pi}$ at $1 / K \simeq 6$ does not change so much. On the other hand, for the massless quark at $1 / K=8$, the pion mass takes a value which is roughly twice the lowest Matsubara frequency, for the both cases of $N_{t}=4$ and 8. The behavior around $1 / K=4$ is similar, but indicates more complicated structure.

\section{4. $K_{d}$ versus $N_{F}$}

We now study the case of $\beta=0$ as in the previous work [1]. Fig. [2 a shows the results of the deconfining transition points $K_{d}$ at $\beta=0$ obtained on $N_{t}=4$ lattices for various numbers of $N_{F}$ (see also Fig. 2b).

The data of plaquette (Fig. 3) for $N_{F}=240$ at $\beta=0$ indicates that the deconfining transition is of first order and that the location of the transition point is independent of $N_{t}$ for large $N_{t}$ 

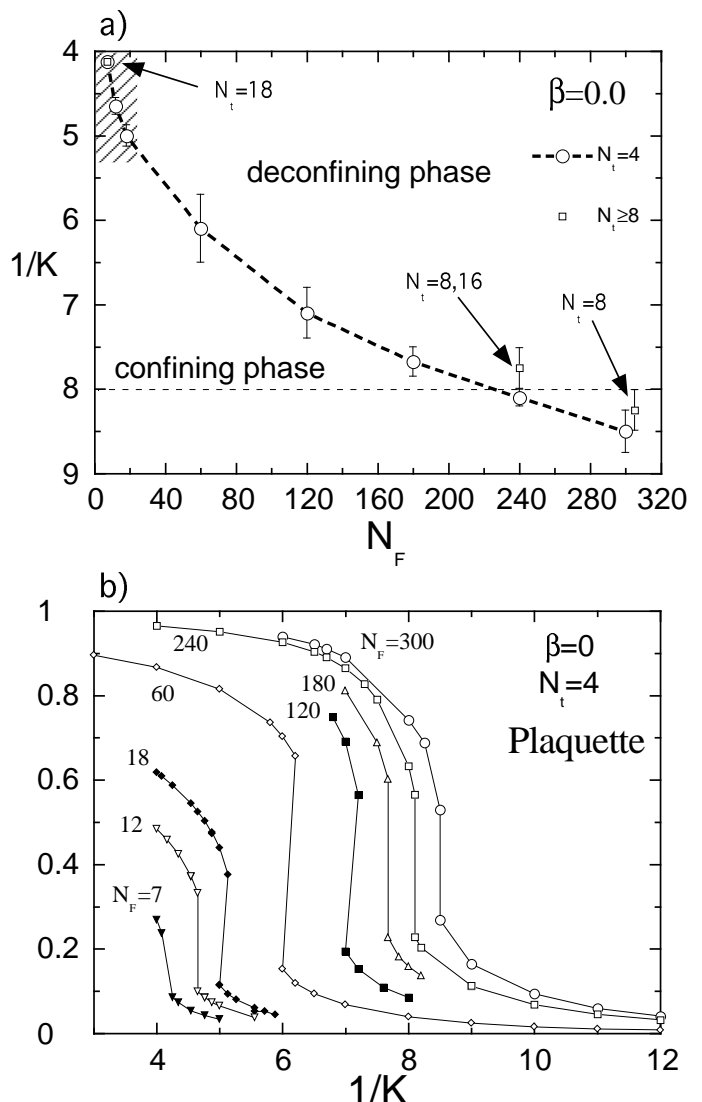

Figure 2. a) The transition point $1 / K_{d}$ at $\beta=0$ versus $N_{F}$ at $N_{t}=4$ and $N_{t} \geq 8$. For clarity, data at $N_{t}=8$ for $N_{F}=300$ is slightly shifted in the figure. Shaded region was investigated in our previous study [1]. b) Plaquette versus $1 / K$ at $\beta=0$ for various number of $N_{F}$ at $N_{t}=4$.

$\left(1 / K_{d} \simeq 8.1\right.$ for $N_{t}=4$ and $1 / K_{d} \simeq 7.75$ for $N_{t}=8$ and 16). Therefore we conclude that the transition is bulk as is confirmed in our previous work for $N_{F}=7$ [1]. In Fig. 2a the transition points at $N_{t} \geq 8$ for $N_{F}=7,240$ and 300 are also included. These values are roughly those for the bulk transition points at zero temperature.

As $N_{F}$ increases up to $240,1 / K_{d}$ approaches toward or exceeds the value 8 , which is the value of $1 / K$ for a massless free quark. Because of this, for $N_{F} \gtrsim 240$, we are able to see a wide region of the deconfining phase in the whole range of $\beta$. Therefore we first intensively investigate the case

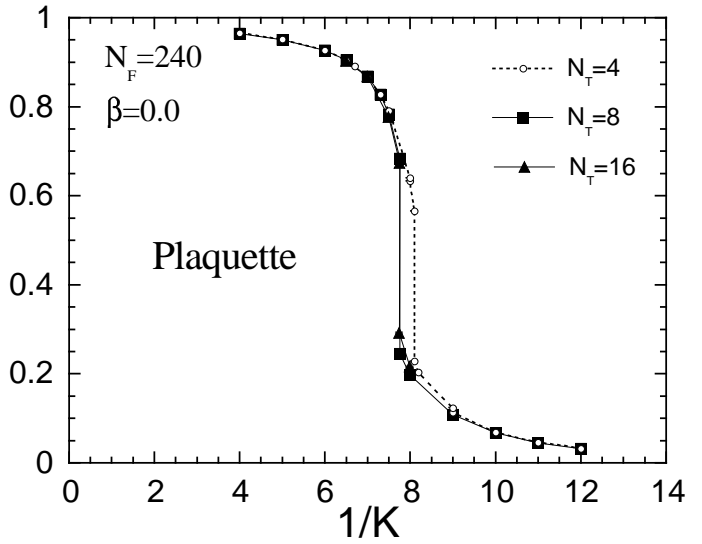

Figure 3. Plaquette at $\beta=0$ for $N_{F}=240$ at $N_{t}=4,8$, and 16 .

$N_{F}=240$, and then decrease $N_{F}$.

5. $N_{F}=240$

Fig. 10 shows the results of $m_{\pi}^{2}$ and $2 m_{q}$ for $N_{F}=240$ at $\beta=0,2.0,4.5,6.0$, and 100 on the $N_{t}=4$ lattice. A very striking fact is that the shape of $m_{\pi}^{2}$ and $2 m_{q}$ as a function of $1 / K$ only slightly changes for $1 / K<8$ when the value of $\beta$ decreases from $\infty$ down 0 . Only the position of the local minimum of $m_{\pi}^{2}$ at $1 / K \simeq 8$, which corresponds to the vanishing point of $m_{q}$, slightly shifts toward smaller $1 / K$. We obtain similar results also for $N_{t}=8$.

From these data we propose the phase diagram in Fig. 固 for $N_{F}=240$. The dark shaded line is the phase boundary between the confining phase and the deconfining phase at zero temperature. When $N_{t}=4$ or 8 , the boundary line bends down at finite $\beta$ as shown in Fig. 5 , due to the finite temperature phase transition of the confining phase.

The dashed line corresponds to the $m_{q}=0$ line. This line also corresponds to the minimum point of $m_{\pi}^{2}$. We have also calculated the quark propagator in the Landau gauge and checked that chiral symmetry of the propagator, $\gamma_{5} G(z) \gamma_{5}=$ $-G(z)$, is actually satisfied on the $m_{q}=0$ line.

The results for the case $N_{F}=300$ are essentially the same as those for $N_{F}=240$ except for very small shifts of the transition point and the 


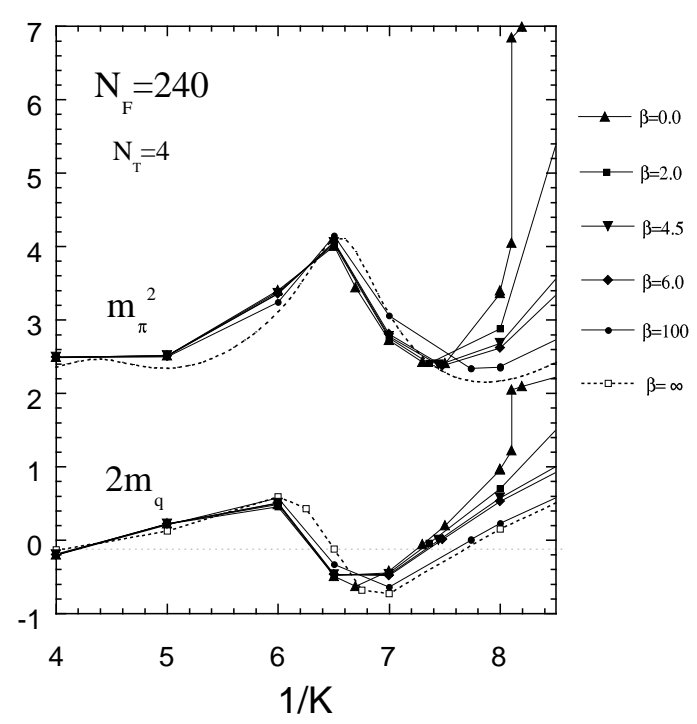

Figure 4. $m_{\pi}^{2}$ and $2 m_{q}$ versus $1 / K$ for $N_{F}=240$ at $N_{t}=4$.

minimum point of $m_{\pi}^{2}$.

\section{Renormalization group flow}

The $m_{q}=0$ point at $\beta=\infty$ is the trivial IR fixed point for $N_{F} \geq 17$. The phase diagram shown in Fig. 5 suggests that there are no other fixed points on the $m_{q}=0$ line at finite $\beta$.

In order to confirm this, we investigate the direction of Renormalization Group (RG) flow along the $m_{q}=0$ line for $N_{F}=240$, using a Monte Carlo Renormalization Group (MCRG) method.

One problem here is that it is practically impossible to make simulations in the massless limit at zero temperature due to the existence of zero modes in the quark matrix. Therefore, we impose an anti-periodic boundary condition in the $t$ direction.

We make a block transformation for a change of scale factor 2 , and estimate the quantity $\Delta \beta$ for the change of $a \rightarrow a^{\prime}=2 a$. We generate configurations on an $8^{4}$ lattice on the $m_{q}=0$ points at $\beta=0$ and 6.0 and make twice blockings. We also generate configurations on a $4^{4}$ lattice and make once a blocking. Then we calculate $\Delta \beta$ by matching the value of the plaquette at each

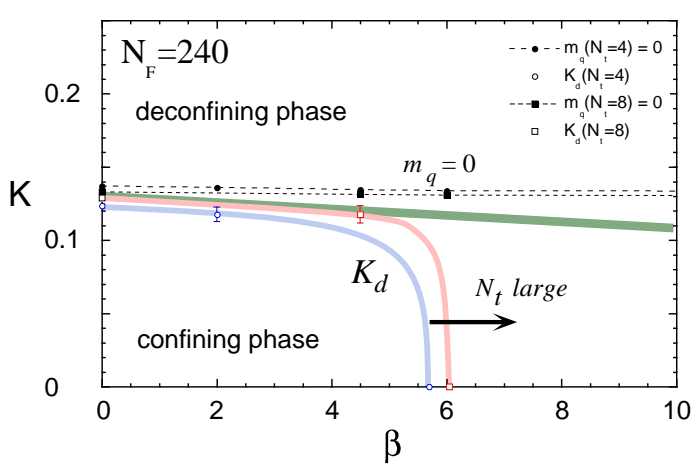

Figure 5. Phase diagram for $N_{F}=240$.

step."

From the matching, we obtain $\Delta \beta \simeq 6.5$ for $\beta=0$ and 10.5 for $\beta=6.0$. The value obtained from the perturbation theory is $\Delta \beta \simeq 8.8$ at $\beta=$ 6.0 for $N_{F}=240$. The signs are the same and the magnitudes are comparable. This suggests that the directions of RG flow on the $m_{q}=0$ line at $\beta=0$ and 6.0 are the same as that at $\beta=\infty$ for $N_{F}=240$. This further suggests that there are no fixed points at finite $\beta$. All of the above imply that the theory is trivial in the case of $N_{F}=240$.

\section{7. $240 \geq N_{F} \geq 17$}

Now we decrease the value of $N_{F}$ from 240 . Fig. 6 shows $m_{\pi}^{2}$ and $2 m_{q}$ for various $N_{F}$, from 300 down to 18 , at $\beta=6.0$ and 0 .

When $\beta=6.0$, the shapes of $m_{\pi}^{2}$ are almost identical to each other, except for a slight shift toward smaller $1 / K$ as $N_{F}$ decreases.

When $\beta=0$, the boundary of the first order phase transition between the deconfining phase and the confining phase moves toward smaller $1 / K$ and therefore the range of the deconfining phase decreases: $1 / K_{d} \simeq 8.5,8,7.6,7.2,6.1$ for $N_{F}=300,240,180,120$ and 60 , respectively, as shown in Fig. 2. For $N_{F}=18$, the value of $1 / K_{d}$ decreases down to 5.0.

Due to the fact that the confining phase invades

1 It is known for the pure $S U(3)$ gauge theory, in particular in the deconfining phase, that one has to make a more careful analysis using several types of Wilson loop to extract a precise value of $\Delta \beta$. We reserve elaboration of this point and a fine tuning of $1 / K$ at each $\beta$ for future works. 


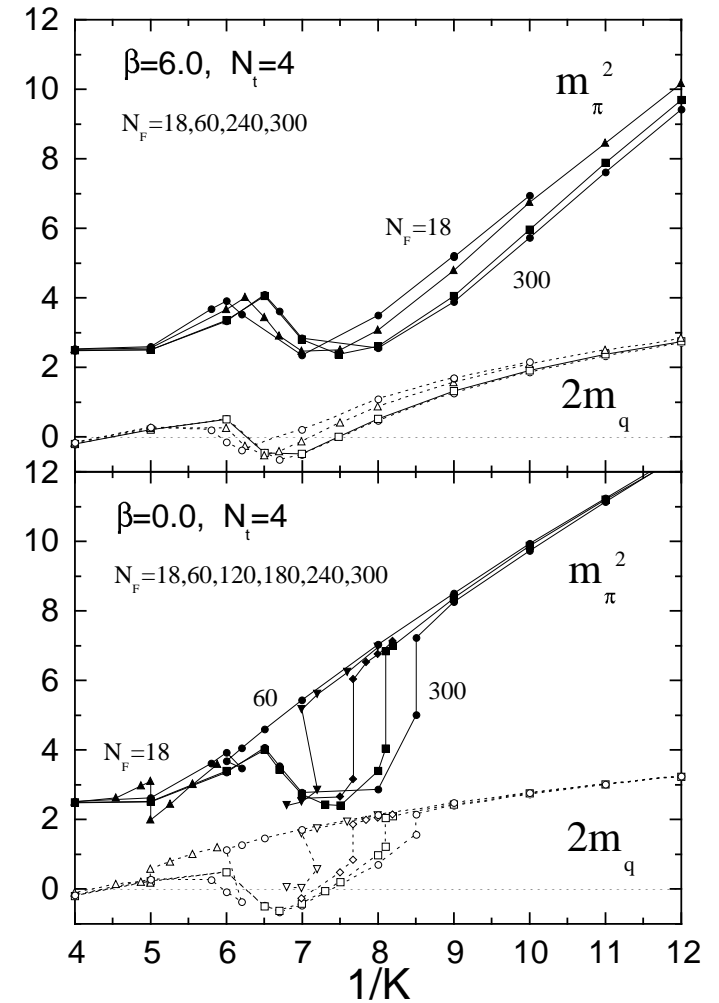

Figure 6. $m_{\pi}^{2}$ and $2 m_{q}$ for $N_{F} \leq 18, N_{t}=4$ at $\beta=6.0$ and 0.0 versus $1 / K$.

the deconfining phase, the massless line in the deconfining phase hits the boundary at finite $\beta$ when $N_{F}$ becomes small. For example, in the case of $N_{F}=18$, as shown in Fig. 7, it hits at $\beta=4.0-4.5$.

Although the area of the deconfining phase decreases with decreasing $N_{F}$, the shape and the position of $m_{\pi}^{2}$ in the part of deconfining phase only slightly change from $N_{F}=300$ to 18 . The values of $m_{q}$ as functions of $1 / K$ also show only slight changes in the deconfining phase. These facts, combined with the perturbative result that for $N_{F} \geq 17, \beta=\infty$ is the IR fixed point, suggest that the RG flow along the massless quark in the deconfining phase is the same as that for $N_{F}=240$. In this case, the theory is trivial for $N_{F} \geq 17$.2

\footnotetext{
${ }^{2}$ Although we have investigated only down to the $N_{F}=$ 18 case, we do not expect any qualitative differences between the cases of $N_{F}=17$ and 18 .
}

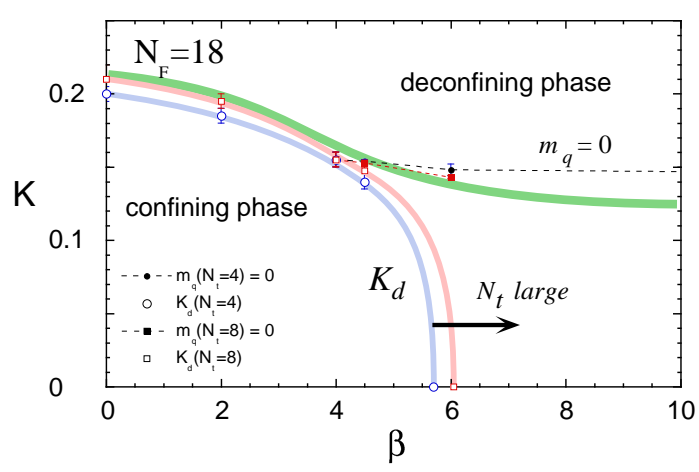

Figure 7. Phase diagram for $N_{F}=18$.

\section{8. $16 \geq N_{F} \geq 7$}

When $N_{F} \leq 16$, the theory is asymptotic free. On the other hand, quark confinement is lost for $N_{F} \geq 7$ even in the strong coupling limit $\beta=0$ [1]. Therefore the question is what happens for the cases $16 \geq N_{F} \geq 7$ in the deconfining phase.

We have intensively simulated the cases $N_{F}=$ 12 and 7 . See the results for the case $N_{F}=7$ in Fig. 8. The gross feature of the phase diagram does not much differ from the case of $N_{F}=18$ which is shown in Fig. 7.

One crucial point is that there is no chiral limit in the confining phase at least for $\beta \lesssim 4.5$. This suggests that quarks are not confined in the continuum limit.

A more subtle point is whether there is a difference between the case $N_{F} \geq 17$ and the case $16 \geq N_{F} \geq 7$ concerning the deconfining phase. For $N_{F} \leq 16$, the massless point at $\beta=\infty$ is a UV fixed point. Therefore the direction of the RG flow around $\beta=\infty$ is opposite to that for $N_{F} \geq 17$. In order to study the phase structure around $\beta=0$, we have performed a MCRG study for the case $N_{F}=12$ similar to that for the case of $N_{F}=240$. We have investigated the RG flow along the maximum of $m_{\pi}^{2}$, because this line roughly corresponds to an infinite quark mass line in a sense explained in Sec. 3. We have found that the RG flow at $\beta=0$ and $\beta=6.0$ are opposite and the flow changes the direction around $\beta=4.0$. This result suggests that there is a fixed point on the $m_{q}=0$ line. The flow of RG on the $m_{q}=0$ line at $\beta=5.0$ is the same as that 
at $\beta=\infty$, which is opposite to that of the case of $N_{F}=240$. This implies that the location of the fixed point is at $\beta<5.0$. Because of the fact that the massless line $m_{q}=0$ hits the boundary between the two phases around $\beta=4.0$, it is difficult to determine the location of the fixed point. Although we have not been able to locate the fixed point, we propose the phase structure, based on the consideration given above, that there is a non-trivial IR fixed point at finite $\beta$, [4] for $16 \geq N_{F} \geq 7$. Of course we have to make a detailed RG study to confirm that there is a non-trivial fixed point, which we reserve for future works. The existence of a non-trivial fixed point implies that the theory in the continuum limit is a non-trivial theory with anomalous dimensions, however, without confinement.

\section{9. $N_{F} \leq 6$}

For $N_{F}=2$ and 3 at finite temperatures, it was shown that $m_{q}$ has an unexpected $1 / K$ dependence in the deconfining phase when $\beta$ is smaller than about 5.3 [5]. The $m_{\pi}^{2}$ as a function of $1 / K$ also shows a cusp. In this section we concentrate on these issues. (For the discussion on the phase structure at zero temperature, see Ref. [8] and references in it.)

We point out that the behaviors of $m_{q}$ and $m_{\pi}$ in the high temperature deconfining phase for $N_{F} \leq 6$ at $\beta \lesssim 5.3$ are similar to those in the deconfining phase at zero temperature for larger $N_{F}$. In Fig. 9, the value of $2 m_{q}$ is plotted vs. $1 / K$ for from $N_{F}=240$ down to $N_{F}=2$ at $\beta=4.5$. Results at $\beta=5.0$ are similar. The value of $2 m_{q}$ for the free quark case is also plotted. There are two major trends when $N_{F}$ decreases from 240 down to 2: One is an overall shift of the function $2 m_{q}$ in the deconfining phase to smaller $1 / K$ and shrinkage of the shape with decreasing $N_{F}$. The other is a shift of the transition point from the confining phase to the deconfining phase toward smaller $1 / K$.

It should be noted that except for the overall shift and shrinkage mentioned above, the shape of the function $2 m_{q}$ in terms of $1 / K$ in the deconfining phase is quite similar for all $N_{F}$ and also similar to that of a free quark. Thus the behavior of $m_{q}$ as well as $m_{\pi}^{2}$ as functions of $1 / K$ in the deconfining phase at $\beta \lesssim 5.3$ can be understood essentially as those of free quarks for $1 / K \leq 8$.

We further note that for $N_{F}=2$ and 3 , the massless quark point (the rightmost $m_{q}=0$ ) in the deconfining phase is different from the $K_{c}$ point in the confining phase for $\beta \lesssim 5.3$. The difference is illustrated in the phase diagram for $N_{F}=2$ on the $(\beta, K)$ plane (see Fig. 10). On the $K_{c}$ line, both $m_{\pi}^{2}$ and $m_{q}$ vanish in the confining phase at zero temperature [8]. The finite temperature transition for $N_{t}=4$ occur on the line $K_{t}$. The massless quark line for $N_{t}=4$ in the high temperature deconfining phase agrees with the $K_{c}$ line at $\beta \simeq \infty$. However, they deviate from each other at $\beta \lesssim 5.3$. This corresponds to the fact that the value of $m_{q}$ does not depend on the phase when $\beta \gtrsim 5.5$, while it strongly depends on the phase when $\beta \lesssim 5.3$ [8,9].

\section{Conclusions}

Based on numerical results combined with the result of the perturbation theory we propose the following picture: There are three categories depending on the number of flavors $N_{F}$ : a free theory for $N_{F} \geq 17$, a non-trivial IR fixed point for $16 \geq N_{F} \geq 7$ and confinement and spontaneous chiral symmetry breaking for $N_{F} \leq 6$. The conclusion that there are three categories depending on $N_{F}$ is in accord with recent theoretical works of $N=1$ super-symmetric QCD [10].

We also find that, for any $N_{F} \geq 7$ and for $N_{F} \leq$ 6 at $\beta \lesssim 5.3$, the $1 / K$ dependences of $m_{q}$ and $m_{\pi}^{2}$ in the deconfining phase are essentially the same as those of free quarks for $1 / K \leq 8$, which show non-trivial structure due to doublers. The peculiar behavior of $m_{q}$ as well as the cusp structure of $m_{\pi}^{2}$ in the deconfining phase at $\beta \lesssim 5.3$ for $N_{F}=2$ 5 9.9. can be understood in terms of the overall shift in $1 / K$ and shrinkage of this structure.

Numerical simulations are performed with HITAC S820/80 at KEK, and Fujitsu VPP500/30 and QCDPAX at the University of Tsukuba. This work is in part supported by the Grants-in-Aid of Ministry of Education, Science and Culture (Nos.07NP0401, 07640375 and 07640376). 


\section{REFERENCES}

1. Y. Iwasaki, K. Kanaya, S. Sakai, and T. Yoshié, Phys. Rev. Lett. 69 (1992) 21.

2. M. Bochicchio et al., Nucl. Phys. B262 (1985) 331.

3. S. Itoh et al., Nucl. Phys. B274 (1986) 33.

4. T. Banks and A. Zaks, Nucl. Phys. B196 (1982) 173.

5. C. Bernard et al., Phys. Rev. D49 (1994) 3574 .

6. C. Bernard et al., Phys. Rev. D46 (1992) 4741; T. Blum et al., ibid. D50 (1994) 3377.

7. Y. Iwasaki et al., Nucl. Phys. B (Proc. Suppl.) $42(1995) 502$.

8. Y. Iwasaki et al., hep-lat/9605030.

9. Y. Iwasaki, Nucl. Phys. B (Proc. Suppl.) 42 (1995) 96; K. Kanaya, ibid. 47 (1996) 144.

10. N. Seiberg, Nucl. Phys. B435 (1995) 129.

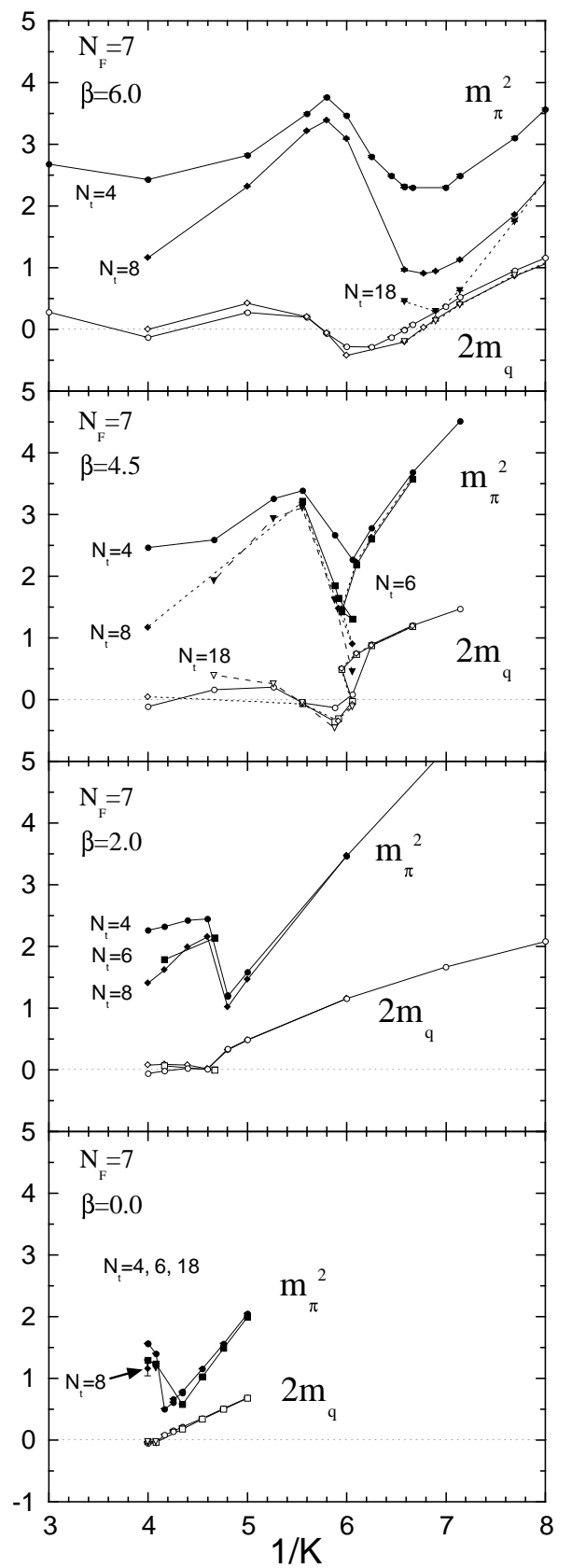

Figure 8. $m_{\pi}^{2}$ and $2 m_{q}$ for $N_{F}=7$ versus $1 / K$. 


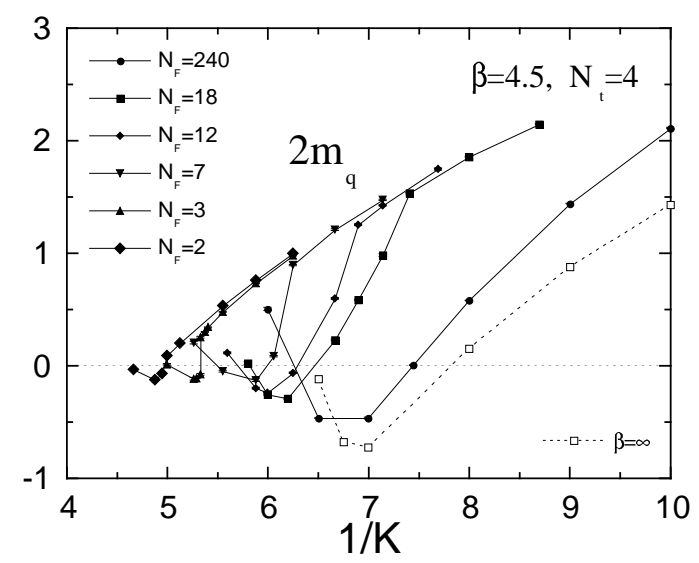

Figure $9.2 m_{q}$ for various number of flavors at $\beta=4.5$ versus $1 / K$.

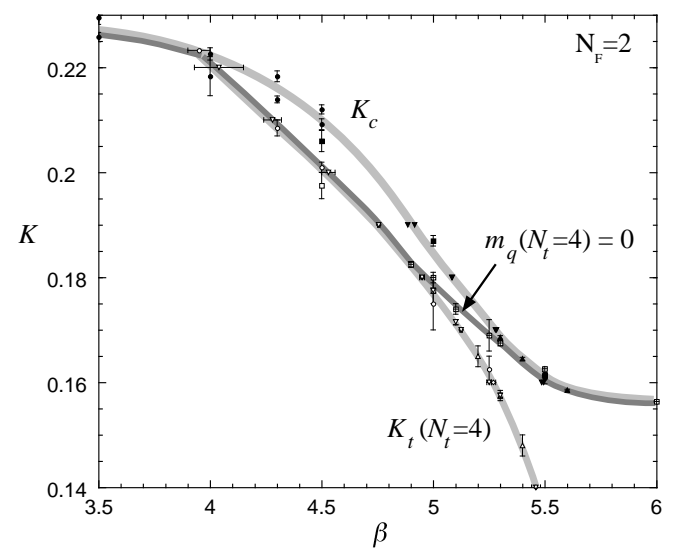

Figure 10. Phase diagram for $N_{F}=2$. 